\title{
Intestinal Amino Acid Absorption in the Fanconi Syndrome: Studies in Patients and in Rats with the Maleic Acid Induced Syndrome
}

\author{
W. NÜTZENADEL, ${ }^{(23)}$ K. FAHR, AND G. HAMMERSEN \\ University of Heidelberg, Children's Hospital Im Neuenheimer Feld 150, Heidelberg, Federal Republic of Germany
}

\section{Summary}

Using a marker perfusion technique, the amino acid absorption in the jejunum from test solutions containing glycine and phenylalanine (30 mM/liter each) was studied in three patients with the Fanconi syndrome. Uptake rates $(\mathrm{mM} / \mathrm{min} / 20-\mathrm{cm}$ seganent) in two cystinotic patients were found to be 55.2 and $\mathbf{7 6 . 1}$ for glycine and 74.5 and 107.5 for phenylalanine. In a patient with idiopathic Fanconi syndrome the rates were 16.5 for glycine and 41.9 for phenylalanine, respectively. If the results are compared to those of healthy controls (74.5 \pm 10.3 for glycine and $107.8 \pm 10.6$ for phenylalanine, respectively) the intestinal absorption of amino acids is normal or slightly decreised in patients with cystinosis but impaired in the latter patient.

Wistar rats treated twice with an injection of maleic acid (3.2 $\mathrm{mM} / \mathrm{kg}$ body weight) were used to study the intestinal absorption in vivo and in vitro. Ten $\mathrm{cm}$ of the jejunum of anaesthetized animals were perfused with a solution consisting of $0.5,1.0$, or $5.0 \mathrm{mM}$ / liter phenylalanine, and the absorptive capacity was calculated from the disappearance of amino acids from the test solutions. The maleic-acid-treated animals exhibited slightly lower rates only at the $5.0 \mathrm{mM} /$ liter concentrations $(P<0.05)$.

The in vitro uptake of phenylalanine and cycloleucine was studied in scraped epithelium of the jejunum. Uptake of both substrates occurs against a concentration gradient, but their transfer was not inhibited by treatment with maleic acid. In contrast, the intracellular accumulation of substrates was found to be higher in the treated animals. Results of these animal studies indicate that maleic acid induces changes of intestinal function, but that inhibition of amino acid absorption does not occur. Comparable to the absorption defect in kidney tubules the Fanconi syndrome may also affect absorptive function in gut mucosa.

\section{FS, Fanconi Syndrome}

\section{Abbreviation}

Fanconi syndrome (FS) consists of a proximal tubular reabsorption defect that involves glucose, amino acids, phosphate, bicarbonate as well as other solutes $(5,21)$. The pathogenesis has not yet been fully elucidated but a noxious effect of an endogenous metabolite or of an exogenous toxic agent has been assumed (21). Similarly, maleic acid injected into rats or dogs causes polyuria, glucosuria, and amino aciduria mimicking human $\operatorname{FS}(2,4,7,9$, 18, 19). Hereditary diseases, such as Hartnup syndrome or cystinuria, indicate a common genetic control and/or function of amino acid transport in epithelium of gut mucosa and kidney tubules, because an impaired transport of an almost similar pattern has been found in both tissues $(1,8,13,21)$.

The question arises whether the noxious effect on renal tubular absorption also affects the absorptive function of gut mucosa. Clinical symptoms do not provide evidence of intestinal involvement, although stunted growth and below average weight are frequently encountered and have been up to now ascribed to the renal dysfunction. But, in hereditary diseases with the combined defect, the impaired absorption in gut mucosa has been neglected for a long time until methods facilitating the measurement of absorption directly at the mucosa surface were developed; moreover, uptake of peptide-linked amino acids compensates for the defective transport of free amino acids. Consequently, the nutritional status of patients with these disorders is not severely affected.

A previously reported investigation (22), studying the absorptive function of gut in rats with the experimentally induced FS, showed an altered sodium and potassium transfer as well as a decreased activity of $\left(\mathrm{Na}^{+}-\mathrm{K}^{+}\right)$ATP-ase; however, amino acid uptake at 1.0 $\mathrm{mM} /$ liter was found to be unaffected. This is surprising because, according to present understanding, sodium flux is an essential feature of epithelial amino acid transport (20); therefore, it seems possible that kinetic differences of amino acid transport have been missed because studies were undertaken at one concentration only.

In order to clarify, whether a defect comparable to that of the kidney tubules exists in the gut mucosa, we have studied the absorption of glycine and phenylalanine in patients with FS using a marker perfusion technique. Additionally, we investigated the intestinal transport of phenylalanine and cycloleucine in rats with an experimentally induced FS.

\section{MATERIALS AND METHODS}

Patients. The upper jejunum of three patients (M.F., 41/2 years and T.M., 51/4 years both with FS due to cystinosis and T.N., 21/2 years with idiopathic FS) was intubated with a double lumen tube. The proximal orifice was placed near the duodeno-jejunal junction whilst the distal opening (distance $20 \mathrm{~cm}$ ) reached the second or third loop of the jejunum. The experiments were carried out after an overnight fast but with the usual supplements of water and electrolytes. The segment was perfused with glycine and phenylalanine, in one patient also with glycyl-phenylalanine $(30 \mathrm{mM} /$ liter, each). The perfusion solution consists also of $\mathrm{NaCl}$ (150 $\mathrm{mM} /$ liter) and polyethylenglycol ( $2 \mathrm{~g} /$ liter). Constant rate perfusion $(5 \mathrm{ml} / \mathrm{min}$ ) was performed by means of a peristaltic pump and the total amount of amino acids perfused was $15 \mathrm{mM} / \mathrm{g}$ body weight. Two samples of intestinal fluid were collected through the tube with the distal opening at $\mathbf{4 0}$ and $50 \mathrm{~min}$.

At the time of the study, urea and creatinin concentrations in plasma were normal in each patient. Parents of the patients were asked for fully informed consent to the study, which was also approved by the local Ethical Committee. Data of controls were taken from a previous published report which also gives more details of the method (15).

Animals. Wistar rats were injected intraperitoneally with a buffered sodium maleate solution $(3.2 \mathrm{mM} / \mathrm{kg}$ body weight; $\mathrm{pH}$, 7.40) on the day before the experiments and $2 \mathrm{~h}$ before the experiments were started. In our experience, only the repeated injections warrented polyuria, as well as glucos- and aminoaciduria in each rat lasting at 5-6 h after the second injection. Control 
animals were injected with saline $(150 \mathrm{mM} /$ liter $)$. Before the experiments, the rats had starved for $16-18 \mathrm{~h}$ with free access to water. Different animals were used for the in vivo and in vitro experiments.

In vivo experiments. Rats (250-350 g) were anaesthetized with thiobarbital (Inactin, BYK-Gulden, Germany, $100 \mathrm{mg} / \mathrm{kg}$ body weight, injected intraperitoneally). Two plastic catheters were inserted into the jejunum, one behind the ligamentum of Treitz, the other $10 \mathrm{~cm}$ more distally. The segment between the cannulae was separated from the remaining gut with two ligatures and the two catheters were led outside the abdominal cavity. After closing the abdominal wall the rats remained unconscious until the end of the experiments. The segment was perfused at a constant rate of $0.04 \mathrm{ml} / \mathrm{min}$ with a solution consisting of either $0.5,1.0$, or 5.0 $\mathrm{mM} /$ liter phenylalanine together with $\mathrm{NaCl}(150 \mathrm{mM} /$ liter) and polyethylenglycol $(2 \mathrm{~g} /$ liter $)$. The order of perfusion of solutes was in sequence of lower to higher concentration in each rat. After 20 min of equilibration the effluent from the distally placed catheter was collected twice over $15 \mathrm{~min}$. The temperature of animals and perfusion solutions was controlled and kept at $37^{\circ} \mathrm{C}$. Previous experiments with perfusion of only one concentration exhibit constant absorption rates in experiments lasting more than $3 \mathrm{~h}$.

In vitro experiments. Everted rings (1 cm width) were prepared from the jejunum after removing the gut of killed animals (180$200 \mathrm{~g}$ ). The rings were incubated with phenylalanine or cycloleucine at various concentrations for $15 \mathrm{~min}$ at $37^{\circ} \mathrm{C}$. Thereafter, the rings were rinsed and blotted on a filter paper and the mucosa was scraped with a metal spatula to remove enterocytes from the submucosa. The enterocyte preparation was weighed on tared aluminum foil before further processing (14). The incubation solution consists of $\mathrm{NaCl}(120 \mathrm{mM} /$ liter $)$, of $\mathrm{KCl}(5.0 \mathrm{mM} / \mathrm{liter})$, of $\mathrm{MgSO}_{4}$ (1.0 mM/liter) of $\mathrm{CaCl}_{2}$ ( $5.0 \mathrm{mM} /$ liter), of Tris (15 $\mathrm{mM} / \mathrm{liter}$ ) and of the labelled and unlabelled amino acids at various concentrations. The solutions were adjusted to $\mathrm{pH} 7.4$ and were gassed with $\mathrm{O}_{2}$ before use and continuously during the experiments.

Analysis and calculations. Amino acid concentrations in the intestinal fluid and plasma were determined by column chromatography on an amino acid analyzer (Biocal BC 202) after deproteinization with trichloracetic acid. Polyethylenglycol was determined according to published methods 10 .

The results of the perfusion experiments are calculated from the disappearance of amino acids from the test solutions as described earlier 15. They are expressed in $\mathrm{mM} / \mathrm{min} /$ segment and are derived from two determinations.

The radioactivity of the enterocyte preparation was extracted by boiling the tissue in $1 \mathrm{ml}$ water, which was added to $10 \mathrm{ml}$ scintillation fluid (Aquasol, New England Nuclear Corp. Boston, MA) and counted in a liquid scintillation counter. The results were calculated from the concentration of free substrate in the intracellular water space after correction for the amount of amino acids in the extracellular space. They are expressed as amount of amino acids per $g$ mucosa or as distribution ratio, which is the ratio of intracellular to extracellular concentration. Uptake velocity and kinetic constants were calculated in the usual manner after transformation of data according to Lineweaver-Burk. Results are derived from four separate determinations in five to six animals (14).

Statistical significance was analyzed by Student's $t$ test.

Materials. L- $\left[\mathrm{U}-{ }^{14} \mathrm{C}\right]$-phenylalanine (specific activity $>450$ $\mathrm{mCi} / \mathrm{mM})$ and 1 -aminocyclopentane- $1-\left[{ }^{4} \mathrm{C}\right]$-carboxylic-acid-cycloleucine (specific activity, $40-60 \mathrm{mCi} / \mathrm{mM}$ ) were purchased from Amersham and Buchler, Germany. Glycine, phenylalanine, and polyethylenglycol (molecular weight, 2000-4000) were obtained from Merck, Germany, and glycyl-phenylalanine from Fluka Buchs, Switzerland. Other chemicals of the highest available purity were obtained from different sources.

\section{RESULTS}

The absorption rates of glycine and phenylalanine from perfusion solutions in gut of patients with FS exhibit a broad range of
Table 1. Intestinal absorption of glycine and phenylalanine in patient with Fanconi syndrome ${ }^{1}$

\begin{tabular}{lccccr}
\hline & \multicolumn{2}{c}{ Absorption rates } & & \multicolumn{2}{c}{$\Delta$ Plasma levels } \\
\cline { 2 - 3 } \cline { 5 - 6 } & gly & phe & & gly & phe \\
\hline Free glycine and phenylalanine & & & \\
T.N. & 16,5 & 41,9 & & 0,098 & 0,291 \\
F.M. & 55,2 & 67,7 & & 0,063 & 0,394 \\
M.T. & 76,1 & 107,8 & & 0,220 & 0,432 \\
Controls & 74,5 & 106,1 & & 0,385 & 0,421 \\
& $\pm 10,3$ & $\pm 10,6$ & & $\pm 0,122$ & $\pm 0,107$ \\
Glycyl-phenylalanine & & & & \\
M.T. & 96,65 & 101,7 & & 0,326 & 0,527 \\
Controls & 110,7 & 113,6 & & 0,356 & 0,432 \\
& $\pm 20,1$ & $\pm 11,1$ & & $\pm 0,091$ & $\pm 0,123$ \\
\hline
\end{tabular}

${ }^{1}$ Absorption rates (mM/min/20-cm segment) are calculated from the disappearance of free and peptide-linked glycine and phenylalanine from test solutions. The increase $(\Delta)$ of these amino acids in plasma (mM/liter) during the perfusion experiments is also shown.

results. Two patients with cystinosis showed absorption rates slightly below or in the range of the controls (Table 1). In contrast, in T.N. suffering from idiopathic FS, the perfusion experiments revealed an absorption rate far below those of the controls. An impaired uptake of glycine and phenylalanine in this patient is also supported by the lower increase of these amino acids in plasma during the perfusion experiment (Table 1).

The absorption of peptide-linked glycine and phenylalanine seems to be unchanged or at least not differently altered than that of free amino acids because patient M.T. showed very similar rates for the uptake of free and peptide-linked amino acids (Table 1).

Controls and patients differ in their age and, probably more importantly, in their body size; thus, the absorbing surface of the perfused segment may vary with different width of gut in patients and controls. But the different absorbing surface does not provide a satisfactory explanation for the findings because the absorption rates of patient M.T. were observed in the range of the controls despite his younger age and smaller body size. With some reservations as to firm conclusions, it seems that the differences are not due to different age, body size, or absorbing surface.

Absorption of amino acids from a $30 \mathrm{mM}$ solution is not comparable to the concentrations that occur during protein digestion in the intestine. With respect to nutritional relevance, experiments with low solute concentrations are desirable. But under those conditions we have experienced problems of precise measurements, which especially hampered the comparison of results between patients and controls. First, the downhill concentration gradient of the solutes studied varies with different absorption capacity, e.g., with perfusion of a $30 \mathrm{mM}$ solution the concentrations of the effluent obtained from the distally placed catheter is $10-15 \mathrm{mM}$ in controls but $18-24 \mathrm{mM}$ in patients with impaired absorption. This difference becomes even more distinct when concentrations of the perfusion solutions are much lower; therefore, the mucosa is likely to be exposed to a different profile of amino acid concentrations along the perfused segment and this may influence the results and their comparison considerably. Second, when concentrations between $0.5-2.5 \mathrm{mM}$ are studied, the levels in intestinal fluid at the site of sampling were found in the range of those samples obtained either during perfusion of saline alone or obtained before the experiments were started. This suggests that under these circumstances the load of amino acids entering the lumen of gut has been totally absorbed before it reaches the sampling site and that equilibrium between absorption and efflux from blood to lumen has been established more proximally. The calculation of absorption rates becomes almost impossible because they are derived from the disappearance of amino acids from test solutions. Last, it is also evident that with the perfusion of low solute concentrations backflux from blood to 
lumen contributes to a greater extent to the concentrations of amino acids in the effluent from which absorption rates are calculated. Considering these factors we chose a $30 \mathrm{mM}$ solute concentration, which is not in excess of the apparent kinetic parameters found in a healthy person (glycine: $\mathrm{km}, 10.0$ and Vmax, 70.2; phenylalanine: $\mathbf{k m}, 18.8$ and $V \max , 107.2)$.

The results of the perfusion experiment in the upper jejunum in rats (Fig. 1) are more consistent. Treated animals exhibit slightly lower uptake but the statistical evaluation of the difference shows only at the $5.0 \mathrm{mM}$ concentration a $P$ value at the 0.05 confidence level. In view of the marked reduction of amino acid absorption in renal tubules after injection of maleic acid, the observed difference seems to be small and does not provide enough evidence of a reduced amino acid uptake in the intestine after maleic acid treatment.

The in vitro technique allows us to study more directly the uptake through the luminal membrane of the enterocyte. Addi-

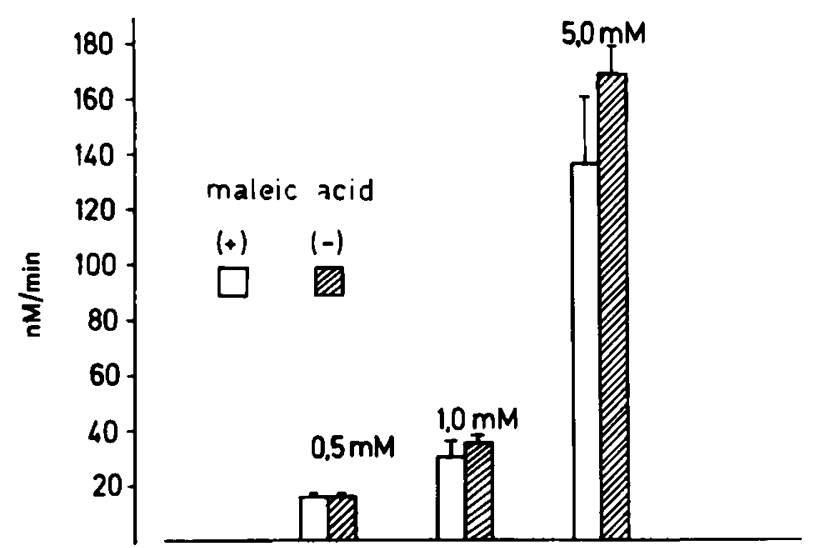

Fig. 1. Intestinal absorption of phenylalanine in rats injected with 3.2 $\mathrm{mM} / \mathrm{kg}$ body weight of maleate (open bars) and of their controls (shaded bars) during perfusion of $10 \mathrm{~cm}$ jejunum. Results are calculated from the disappearance rates from test solutions containing either $0.5,1.0$, or 5.0 $\mathrm{mM} /$ liter phenylalanine. Differences of treated and untreated animals are only valid at the $5.0 \mathrm{mM} /$ liter concentration $(P=0.05)$. tionally, a broad range of substrate concentrations can easily be investigated without the influence of water secretion or of endogenous amino acids delivered into the lumen of the gut.

As shown in Figure 2, no inhibition of amino acid uptake was observed. In contrast, the mucosa of the treated animals accumulated more of the amino acids tested. Due to the variation of findings between the animals, the uptake rates at each concentration are, with few exceptions, not statistically different. But, if the results of the distribution ratio of all concentrations are combined and evaluated in a paired fashion the differences between treated and untreated rats are statistically valid with a $P$ value $<0.01$ for both amino acids studied.

It is noteworthy, that with the mucosa preparation used phenylalanine as well as cycloleucine are concentrated in the tissue against a gradient with a distribution ratio of the isotope between 1.5-10. Further, the uptake up to $30 \mathrm{~min}$ is linear. Accordingly, at 15 min incubation time, we observed mainly initial uptake with a flow direction into the enterocytes.

A profound effect of maleic acid on cellular metabolism has been described $(9,16)$, but it seems very unlikely that maleic acid affects metabolism of the amino acid used since degradation of phenylalanine in the enterocytes is low and it does not occur with cycloleucine.

\section{DISCUSSION}

The results of the study do not allow a definite answer to the question: does FS also cause an impaired amino acid absorption in the intestine as it does in renal tubules? It seems that there is no clear answer because findings in patients are not uniform. In our opinion the results should not be regarded as unreliable or due to experimental variations. The perfusion technique is one of the most accurate methods to study absorption because the influence of gastric emptying, transit time, and endogenous metabolism is excluded. In a previous investigation it was proven as a sensitive method to discover impaired absorptive function 15 . We were more concerned about the different body size of patients and controls, but to perform the experiments in younger healthy probands who could not volunteer did not seem justified to us. On the other hand, the broad variation of absorption rates in patients with small but very similar body size and the narrow
PHENYLALANINE

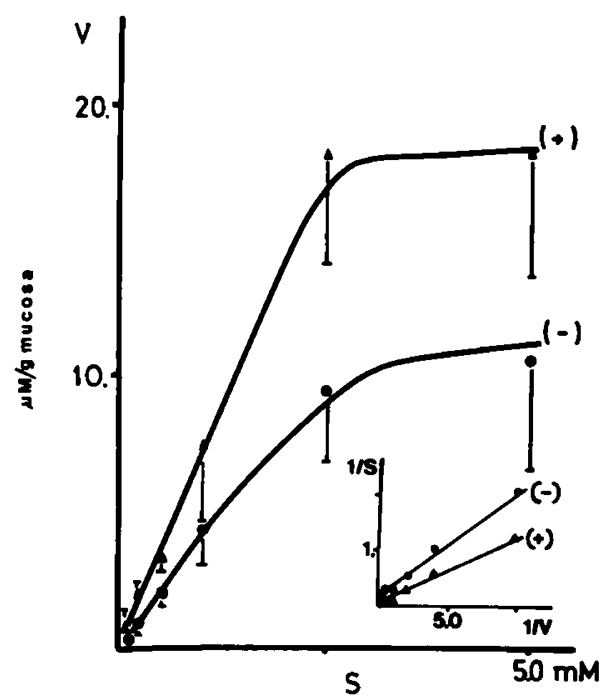

\section{CYCLOLEUCINE}

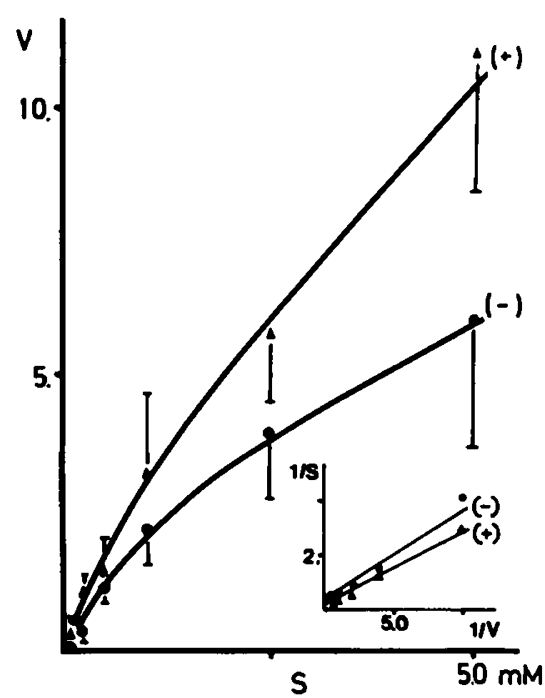

Fig. 2. Uptake of phenylalanine and cycloleucine into enterocytes of rats with the maleic acid-induced Fanconi syndrome and their controls. The accumulation of substrates are higher in treated $(+)$ than untreated $(-)$ animals but significant differences $(P \leq 0.05)$ are calculated only for the comparison of results at $0.05-0.5 \mathrm{mM} /$ liter phenylalanine concentrations. The inserts show the calculated regression of the transformed data according to Lineweaver-Burk. From these data the "apparent" kinetic parameters are derived. Phenylalanine uptake after treatment: Vmax, 10.8 and $\mathrm{Km}, 2.23$ $\mathrm{mM}$ compared with controls: Vmax, 10.8 and $\mathrm{Km}, 1.60 \mathrm{mM}$. Cycloleucine uptake after treatment: Vmax, 10.8 and $\mathrm{Km}, 2.91 \mathrm{mM}$ compared with controls: $V \max , 4.82$ and $\mathrm{Km}, 1.54 \mathrm{mM}$. The $V \max$ data are expressed as $\mu \mathrm{M} \cdot \mathrm{g}$ mucosa ${ }^{-1} \cdot 15 \mathrm{~min}^{-1}$ incubation time. 
range in the controls do not point to a great influence of either height, weight, or age on intestinal absorptive function.

Keeping in mind that FS has no uniform pathogenesis, and that even its expression of renal symptoms varies, it is likely to assume that FS does not impair intestinal function of patients with cystinosis but it may affect the intestinal mucosa of patients with other causes of FS. This discordance of a renal tubular and an intestinal mucosal absorption defect has already been demonstrated in a patient with FS due to Lowe's syndrome (3). In this patient the mucosal uptake in intestine was found to be decreased for dibasic amino acids but normal for glycine, whereas the renal tubular absorption defect involves dibasic as well as neutral amino acids. In this context, it is also noteworthy that alterations of intestinal cellular morphology in cystinosis are more or less confined to the reticuloendothelial cells of lamina propria. Biopsy findings of two patients reported in the literature $(11,12)$ and of our patient M.T. revealed an almost normal appearance of the epithelium. Similarly, the activity of brush-border disaccharidases of the latter patient was in the normal raige. It would be of high interest to have observations on morphology and other intestinal functions in the patient with the impaired amino acid absorption. But we could not obtain permission to perform an intestinal biopsy.

The results of the in vivo experiments in rats agree with published data studying amino acids uptake from perfused segments (22). At 0.5 and $1.0 \mathrm{mM}$ phenylalanine concentrations no influence of maleic acid on amino acid absorption was found. The difference at $5.0 \mathrm{mM}$ concentration is small and is due possibly to an experimental variation rather than a true effect of the treatment. With respect to the nutritional relevance, an absorptive defect involving only high amino acid concentration in the intestinal fluid would be of little or no importance.

The in vitro experiments show unexpected results because uptake of phenylalanine and cycloleucine is higher in treated animals. Though the differences at single concentrations tested are small, statistical validity is only given if the results of all concentrations are compared in a paired fashion. The uniform trend at each concentration of both amino acids makes experimental error rather unlikely.

The exact site and the mode of maleic acid action on renal tubular function has not been clarified, but the results of published investigations suggest three possibilities: (1) derangement of energy utilization required for active transport; (2) alteration of membrane permeability either at luminal or basolateral site of the epithelial cells; and (3) interference of maleic acid with carrier molecules responsible for the transport of solutes into the cells (6).

Work with isolated brush border membranes of rat kidney showed no effect of maleic acid on transport into the vesicles excluding more or less the last possibility (17). But a disturbed energy utilization may alter structural or functional properties of the membrane, which may not only inhibit but enhance transfer of solutes through the cell membrane $(4,9)$; moreover, the transepithelial transport involves several steps and possibly different pathways on which the effect of maleic acid could be opposit. Assuming a more complex action the results of investigations depend very much on the choosen experimental condition, and an enhanced influx into the cells may also be observed.

In general, the animal studies exclude the possibility of an inhibited amino acid transport through the action of maleic acid but indicate an impairment of other intestinal functions. This conclusion is also supported by the published findings of an altered sodium and potassium transport but an unaffected absorption of amino acids in intestine of rats after treatment with maleate (22).

\section{REFERENCES AND NOTES}

1. Asatoor, A. M., Cheng, B., Edwards, K. D. G., Lant, A. F., Matthews, D. M., Milne, H. D., Navab, F., and Richards, A. J.: Intestinal absorption of two didpetides in Hartnup disease. Gut, 11: 380 (1970).

2. Asatoor, A. M., Bending, M. R., and Milne, M. D.: Peptiduria in experimental Fanconi syndrome in rats. Clin. Sci., 57: 277 (1979).

3. Bartsocas, Ch. S., Levy, H. L., Crawford, J. D., and Thier, S. O.: A defect in intestinal amino acid transport in Lowe's syndrome. Am. J. Dis. Child., 117: 93 (1969).

4. Bergeron, M., Dubord, L., and Hauser, C.: Membrane permeability as a cause of transport defects in experimental Fanconi syndrome. J. Clin. Invest., 57: 1181 (1976).

5. Fanconi, G.: Der frühinfantile nephrotisch-glucosurische Zwergwuchs mit hypophosphatämischer Rachitis. Jhrb. Kinderheilk., 147: 299 (1936).

6. Günther, R., Silbernagel, S., and Dettjen, P.: Maleic acid induced aminoaciduria, studied by free flow micropuncture and continuous microperfusion. Pflügers Arch., 382: 109 (1978).

7. Harrison, H. E. and Harrison, H. C.: Experimental production of renal glucosuria, phosphaturia, and aminoaciduria by injection of maleic acid. Science, 120:606 (1954).

8. Hellier, M. D., Holdworth, C. D., Perrett, D., and Thirum Rai, C.: Intestinal transport in normal and cystinuric subjects. Clin. Sci., 43: 659 (1972).

9. Kramer, H. J. and Gonick, H. C.: Experimental Fanconi syndrome: effect of maleic acid on renal cortical Na-K-ATPase activity, and ATP levels. J. Lab. Clin. Med., 76: 799 (1970).

10. Malawer, S. J. and Powell, D. W.: An improved turbidometric analysis of polyethylenglycol utilizing an emulsifier. Gastroenterology, 65: 192 (1967).

11. Meyer, M., Palcoux, J. B., Lagarde, N., and Walter, S.: Exploration digestive de la cystinose. Arch. Fr. Pediatr., 37: 607 (1980).

12. Morecki, R., Paunier, L., and Hamilton, J. R.: Intestinal mucosa in cystinosis. Arch. Pathol., 86: 297 (1968).

13. Navab, F. and Asatoor, A. M.: Studies on intestinal absorption of amino acids and a dipeptide in a case of Hartnup disease. Gut, 11: 373 (1970).

14. Nützenadel, W. and Scriver, C. R.: Uptake and metabolism of $\beta$-alanine and Lcarnosine by rat tissues in vitro: role in nutrition. Am. J. Physiol., 230: 643 (1976).

15. Nützenadel, W., Fahr, K., and Lutz, P.: Absorption of free and peptide-linked glycine and phenylalanine in children with celiac disease. Pediatr. Res., 15: 309 (1981).

16. Ragulski, J. and Angielski, S.: Effect of maleic acid on the kidney IV: Synthesis of amino acids in the kidney of maleate treated rats. Acta Biochem. Pol., 10: 133 (1963).

17. Reynolds, R., Mc Namara, P. D., and Segal, S.: On the maleic acid induced syndrome: Effects on transport by isolated rat kidney brush border membrane vesicles. Life Sci., 22: 39 (1978).

18. Rosen, W. J., Kramer, H. J., and Gonick, H. C.: Experimental Fanconi syndrome: Effect of maleic acid on renal tubular ultrastructure. Lab. Invest., 28: 446 (1973).

19. Rosenberg, L. E. and Segal, S.: Maleic acid induced inhibition of amino acid transport in rat kidney. Biochem. J., 92: 345 (1964).

20. Schultz, S. G.: Transport across small intestine. In "Membrane Transport in Biology". Giebisch, G., Tosteron, D. C., and Ussing, H. H. Eds., p. 772. (Springer, Berlin 1979).

21. Scriver, C. R. and Rosenberg, L. E.: Generalized disorders of amino acid transport: The Fanconi syndrome. In Amino Acid Metabolism and its Disorder. Scriver, C. R., and Rosenberg Eds., p. 197. (W. B. Saunders, Philadelphia 1973).

22. Wapnir, R. A., Exeni, R. A., McViar, M., De Rosar, F. J., and Lifshitz, F.: Inhibition of sodium intestinal transport and mucosal $\left(\mathrm{Na}^{+} \mathrm{K}^{+}\right)$-ATPase in experimental Fanconi syndrome. Proc. Soc. Exp. Biol. Med., 150: 517 (1975),

23. Requests for reprints should be addressed to: Dr. W. Nützenadel, Children's Hospital, University of Heidelberg, Im Neuenheimer Feld 150, 6900 Heidelberg, Germany.

24. The research was supported by a grant of Deutsche Forschungsgemeinschaft.

25. Received for publication May 11, 1982.

26. Accepted for publication February 7, 1983. 